\title{
Olfactory Deficits in Patients With Mild Cognitive Impairment Predict Alzheimer's Disease at Follow-Up
}

D.P. Devanand, M.D.

Kristin S. Michaels-Marston, M.A.

Xinhua Liu, Ph.D.

Gregory H. Pelton, M.D.

Margarita Padilla, M.A.

Karen Marder, M.D.

Karen Bell, M.D.

Yaakov Stern, Ph.D.

Richard Mayeux, M.D., M.Sc.

\begin{abstract}
Objective: This study evaluated the predictive utility of olfactory identification deficits in patients with mild cognitive impairment for follow-up diagnosis of probable Alzheimer's disease.
\end{abstract}

Method: Ninety outpatients with mild cognitive impairment were examined at 6-month intervals. Matched healthy comparison subjects $(\mathrm{N}=45)$ were examined annually. The University of Pennsylvania Smell Identification Test was given at baseline.

Results: Olfaction scores were lower in patients with mild cognitive impairment than in healthy comparison subjects. Seventy-seven patients were followed up; 19 were diagnosed with Alzheimer's disease by 2 years. Patients with low olfaction scores ( $\leq 34$ of 40 ), and patients with low olfaction scores who reported no subjective problems smelling, were more likely to develop Alzheimer's disease than other patients. In a Cox proportional hazards model adjusted for age, sex, modified Mini-Mental State score, and education, low olfaction scores did not predict time until development of Alzheimer's disease, but low olfaction scores accompanied by lack of awareness of olfactory deficits predicted time to development of Alzheimer's disease. This effect remained when attention or memory measures replaced modified Mini-Mental State score in the model. In patients with high Mini-Mental State scores ( $\geq 27$ of 30 ), low olfaction with lack of awareness remained a significant predictor of Alzheimer's disease. Olfaction scores of 30-35 showed moderate to strong sensitivity and specificity for diagnosis of Alzheimer's disease at follow-up.

Conclusions: In patients with mild cognitive impairment, olfactory identification deficits, particularly with lack of awareness of olfactory deficits, may have clinical utility as an early diagnostic marker for Alzheimer's disease.
$\mathrm{E}$ tion occurs in the entorhinal-hippocampal-subicular complex, and the neurons of the olfactory epithelium show numerous neurofibrillary tangles (1). Studies of olfactory tasks have consistently shown olfactory identification (discrimination among odors) deficits in patients with Alzheimer's disease in relation to comparison subjects (2-5). There is some evidence that the severity of olfactory dysfunction increases with age (6) and severity of dementia $(3,7,8)$. Impaired olfactory detection has been observed in first-degree relatives of patients with Alzheimer's disease (9). The association between olfactory dysfunction and Alzheimer's disease shows moderate specificity against common neurologic and psychiatric disorders (10). Deficits in olfactory identification are not consistently seen in patients with Huntington's disease (11) or depression (12), but mild deficits have been observed in the Parkinson's dementia complex of Guam (11) and in amyotrophic lateral sclerosis (13), whereas the findings for Parkinson's disease are equivocal (14).

There is limited information on olfactory identification test performance in patients with mild cognitive impairment, defined broadly as fitting into the category between being "normal" and having "dementia." Nordin and Mur- phy (5) have shown that compared to 16 normal comparison subjects, 16 patients with "questionable" Alzheimer's disease had significantly higher thresholds for odor but not for taste, performed significantly worse on tests of recognition memory for odors and visual stimuli, and tended to be less familiar with odors but not visual stimuli.

Early detection of Alzheimer's disease has clinical and potential therapeutic application in patients with mild cognitive impairment, some of whom progress to Alzheimer's disease, while others do not (15). Lack of awareness of cognitive deficits is common in Alzheimer's disease and may occur at the mild cognitive impairment stage, before the diagnosis of Alzheimer's disease is made (16). Similarly, lack of awareness of olfactory identification deficits may occur in patients with mild cognitive impairment, which, to our knowledge, has not been studied previously. We hypothesized that olfactory identification deficits, particularly when accompanied by lack of awareness of olfactory deficits, characterize patients with mild cognitive impairment who subsequently develop Alzheimer's disease. We report initial findings to test these hypotheses in a study of patients with mild cognitive impairment (and normal comparison subjects) followed systematically in a clinical setting. 


\section{Method}

\section{Subjects}

Outpatients with mild cognitive impairment who were seen at a memory disorders center were recruited for a prospective study that examined putative early diagnostic markers of Alzheimer's disease. The research protocol was approved by the institutional review boards of the New York State Psychiatric Institute and Columbia Presbyterian Medical Center, and written informed consent was obtained from each patient. Patients were examined at 6-month intervals. Normal comparison subjects, who were matched to the patients with mild cognitive impairment on age, sex, and years of education, were examined annually.

For patients, inclusion criteria were age $\geq 40$ years, intellectual impairment for $\geq 6$ months and $\leq 10$ years, and the diagnosis of "not demented" (score $=0$ ) or "questionably demented" (score $=$ 0.5) on the Clinical Dementia Rating scale (17). Patients had a minimum modified Mini-Mental State score of $\geq 40$ of 57 (equivalent to Mini-Mental State score of $\geq 22[18,19]$ ), with the caveat that primarily Spanish-speaking patients with $\leq 5$ years of education were included if they had a modified Mini-Mental State score of $\geq 35$. The following deficits on neuropsychological testing served as screening guidelines: impairment in memory, as evidenced by recall of fewer than three of three objects after 5 minutes on the modified Mini-Mental State (18), a delayed recall score of more than one standard deviation below norms in the six-trial Selective Reminding Test (20), or impaired intellectual performance, as evidenced by a WAIS-R performance IQ $\geq 10$ points below the WAIS-R verbal IQ. These neuropsychological criteria served only as screening guidelines; the final determination for study inclusion was based on a consensus diagnosis by expert raters.

Exclusion criteria were a diagnosis of dementia, schizophrenia, schizoaffective disorder, or primary major affective disorder that clearly preceded the onset of cognitive impairment; ECT within the past 6 months; current or recent (past 6 months) history of alcohol or substance dependence (per DSM-IV criteria); clinical or historical evidence of stroke (cortical stroke or an infarct $\geq 2 \mathrm{~cm}$ in diameter on any magnetic resonance imaging [MRI] slice; periventricular hyperintensities and small subcortical lacunae or infarcts did not lead to exclusion); cognitive impairment rated as entirely caused by concomitant medications; and the presence of major neurologic illness (e.g., Parkinson's disease or amyotrophic lateral sclerosis). These inclusion and exclusion criteria defined a relatively broad group of patients between "normal" and "with dementia" and were similar to those used in other studies of mild cognitive impairment $(21,22)$.

\section{Procedures}

At the initial evaluation, a study physician (neurologist or psychiatrist) obtained a detailed medical history and conducted general physical, neurological, and psychiatric examinations. Laboratory tests included a CBC with differential and measures of serum electrolytes, liver and renal function, thyroid function, VDRL, serum $B_{12}$ and folate levels, and an MRI scan of the brain. A trained neuropsychology technician administered the following tests: the WAIS-R, the Wechsler Memory Scale (23), the Selective Reminding Test (24), the Rosen Drawing Test (25), the Controlled Oral Word Association Test (26), the category naming test from the Boston Diagnostic Aphasia Evaluation (27), the Boston Naming Test (28), the Benton Visual Retention Test (29), and the Target Finding Test (30) (shape and letter cancellation tasks). A senior neuropsychologist (Y.S.) reviewed the test results, and a diagnostic impression and report were completed for each patient.

A team of neurologists, psychiatrists, and neuropsychologists, including the evaluating physician, reviewed all the available in- formation and reached a provisional diagnosis. Subsequently, two authors who were also expert clinical raters (D.P.D. and Y.S.) used this information and additional data (e.g., the patient's report or behavior during testing procedures) to make independent diagnoses, followed by a consensus research diagnosis. This consensus diagnosis, made by the two raters, determined study inclusion or exclusion.

At annual follow-ups, a similar set of evaluations was conducted. Only the information obtained from those visits was available, from which the two raters independently made diagnoses. If the diagnosis changed from the previous evaluation, a review of the entire medical and research record was conducted to confirm the change. A diagnosis of dementia was made on the basis of DSM-IV criteria, and the diagnosis of possible or probable Alzheimer's disease was made on the basis of criteria from the National Institute of Neurological and Communicative Disorders and Stroke and the Alzheimer's Disease and Related Disorders Association (31). For the diagnoses of both cognitive impairment and dementia, the most likely etiology (or etiologies) was identified. The consensus diagnosis made by the two expert raters was the primary outcome variable. There was diagnostic agreement in 95.5\% of the cases for the two expert raters' independent diagnosis of dementia versus no dementia.

At baseline evaluation, with the use of a rating on a 4-point scale for lack of awareness of cognitive impairment (32), 16 of the 19 patients who developed Alzheimer's disease on follow-up scored 1 (fully aware of cognitive impairment), and three patients scored 2 (mild lack of awareness of cognitive impairment). This near-universal high level of awareness of cognitive impairment was likely due to inherent bias resulting from the study group, which comprised patients with mild cognitive impairment who brought themselves to a memory disorders clinic for evaluation; hence, this variable was not analyzed as a predictor of Alzheimer's disease at follow-up.

At initial evaluation donepezil had been taken by one patient, vitamin $\mathrm{E}$ or ginkgo biloba by 10 patients, and estrogen by seven patients. The use of these putative cognitive enhancers was analyzed as a dichotomous variable (present or absent). At follow-up one patient had taken donepezil, three to nine patients had taken vitamin E or ginkgo biloba, and three to eight patients had taken estrogen at various times.

For patients with mild cognitive impairment and comparison subjects, apolipoprotein E genotyping was conducted by means of standard methods in which DNA was amplified by the polymerase chain reaction (33). Genotypes were determined while researchers were blind to subject status (patient or comparison) by the sizes of DNA fragments present and viewed and photographed under ultraviolet light after staining with $0.5 \mu \mathrm{g}$ of ethidium bromide.

\section{Test of Olfaction}

Strong psychometric properties have been demonstrated for the University of Pennsylvania Smell Identification Test (34), a scratch-and-sniff test that takes 10-15 minutes to administer. Each of 40 common odorants is embedded in a microcapsule on a separate page, and the subject has to select one of four written multiple-choice alternatives for each odorant (total score range= $0-40$ ). At the initial evaluation a trained research coordinator administered the University of Pennsylvania Smell Identification Test to patients with mild cognitive impairment and comparison subjects, presenting stimuli to both nostrils simultaneously. The total score obtained for each subject was used in statistical analyses. In the University of Pennsylvania Smell Identification Test, introductory questions requested a smoking history, and the derived smoking variables (current or past smoking history) were also evaluated. 


\section{Statistical Analyses}

Two-tailed t tests or chi-square analyses were conducted to compare the demographic and clinical features and olfaction scores of patients with mild cognitive impairment and normal comparison subjects. Two-tailed t tests or chi-square analyses were also conducted to evaluate the association between patients with and without the follow-up diagnosis of dementia and baseline age, sex, modified Mini-Mental State score, years of education, and olfaction scores. The initial question on the University of Pennsylvania Smell Identification Test states, "Do you suffer from smell problems?" Possible answers are "yes" or "no." Patients with mild cognitive impairment who had low baseline olfaction scores $(\leq 34$; cutoff point was the median score of patients with mild cognitive impairment who did not develop Alzheimer's disease by follow-up) accompanied by the report of no problems smelling were classified into one group and the remainder of the patients made up the other group. This dichotomous classification variable, "low olfaction plus lack of awareness," was analyzed in addition to total olfaction score on the University of Pennsylvania Smell Identification Test.

Because of varying lengths of follow-up, survival analysis was the main statistical method employed. A Kaplan-Meier nonparametric survival function was calculated. A global test for proportions was used to check the assumptions of the Cox proportional hazards model, which was used to assess the relative risk of incident Alzheimer's disease. The timing variable was the time from the initial visit to the first follow-up, during which a diagnosis of Alzheimer's disease was made. In addition to the two olfaction measures, age, sex, modified Mini-Mental State score, and years of education were included as variables in these analyses. The choice of these covariates was on based on their association with the outcome of Alzheimer's disease in this clinical group, which was consistent with the literature $(21,22)$. To evaluate prediction of Alzheimer's disease in the intermediate term, logistic regression analyses were conducted with the same covariates after restricting the group with mild cognitive impairment to patients who had completed the 2-year follow-up (or developed Alzheimer's disease before that time); the binary outcome was the indication of developing Alzheimer's disease within 2 years of the baseline evaluation. Sensitivity and specificity for the follow-up diagnosis of Alzheimer's disease were calculated for both low olfaction and the low olfaction plus lack of awareness variable across a range of scores (27-37) on the University of Pennsylvania Smell Identification Test for the group followed up at 2 years.

\section{Results}

\section{Baseline Demographic and Clinical Features}

The study clinical group comprised 90 patients with mild cognitive impairment ( 47 women, $52.2 \%$ ) and 45 normal comparison subjects ( 24 women, 53.3\%). At the initial evaluation patients with mild cognitive impairment had a mean age of 66.7 years $(\mathrm{SD}=10.7)$ and a mean of 15.0 years of education ( $\mathrm{SD}=3.9)$. For normal comparison subjects, the mean age was 64.0 years $(S D=10.0)$, and the mean number of years of education was $15.6(\mathrm{SD}=2.6)$. The mean modified Mini-Mental State score (range $=0-57$ ) was significantly lower in patients (mean $=51.3, \mathrm{SD}=4.4)$ than in comparison subjects (mean $55.0, \mathrm{SD}=1.5)(\mathrm{t}=4.9, \mathrm{df}=$ $133, \mathrm{p}<0.001$ ), as was the mean Mini-Mental State score (patients: $27.4, \mathrm{SD}=2.1$; comparison subjects: $29.4, \mathrm{SD}=$ $0.8)(\mathrm{t}=4.5, \mathrm{df}=133, \mathrm{p}<0.001)$. In the 90 patients with mild cognitive impairment, the clinical dementia rating was 0 (no dementia) in 38.9\% $(\mathrm{N}=35)$ and 0.5 (questionable dementia) in $61.1 \%(\mathrm{~N}=55)$.

\section{Olfaction at Initial Evaluation}

The mean baseline olfaction total score was significantly lower in patients with mild cognitive impairment (31.0, $\mathrm{SD}=7.4)$ than in normal comparison subjects $(35.2, \mathrm{SD}=$ 3.9) ( $\mathrm{t}=3.6, \mathrm{df}=133, \mathrm{p}<0.001)$. In patients, baseline olfaction scores correlated inversely with age (Pearson's $\mathrm{r}=-0.44, \mathrm{df}=$ $88, \mathrm{p}<0.001$ ), positively with baseline modified Mini-Mental State score $(\mathrm{r}=0.37, \mathrm{df}=88, \mathrm{p}<0.001)$, and positively with years of education $(\mathrm{r}=0.27, \mathrm{df}=88, \mathrm{p}<0.02)$ but not with sex, duration of cognitive impairment, score on the Blessed Functional Activity Scale (35), or current or past smoking history. In normal comparison subjects, the mean baseline olfaction score showed no significant associations with age, sex, years of education, current or past smoking history, or modified Mini-Mental State score.

\section{Follow-Up Evaluation}

In the 77 patients with mild cognitive impairment who were followed up (13 recently recruited patients had not yet returned for follow-up), the mean duration of followup was 20 months $(S D=12)$. Of these 77 patients, all 19 who met the consensus diagnostic criteria for dementia also met the criteria for probable Alzheimer's disease (31). The patients who used putative cognitive enhancers were not significantly less likely to develop Alzheimer's disease than the remainder of the clinical group.

\section{Prediction of Alzheimer's Disease at Follow-Up}

Women were more likely than men to receive a final diagnosis of Alzheimer's disease $\left(\chi^{2}=4.8, \mathrm{df}=1, \mathrm{p}<0.05\right)$. Age $(\mathrm{t}=3.2, \mathrm{df}=75, \mathrm{p}<0.01)$, fewer years of education $(\mathrm{t}=2.4, \mathrm{df}=$ $75, \mathrm{p}<0.05)$, low baseline modified Mini-Mental State scores $(\mathrm{t}=3.1, \mathrm{df}=75, \mathrm{p}<0.01)$, and low baseline olfaction scores $(\mathrm{t}=3.4, \mathrm{df}=75, \mathrm{p}<0.001)$ were each associated with the diagnosis of Alzheimer's disease at follow-up. Family history of dementia and baseline scores on the Blessed Functional Activity Scale and the 17-item Hamilton Depression Rating Scale were not associated with a final diagnosis of Alzheimer's disease. Sixteen of 64 patients who reported no problems smelling, compared to three of 13 patients who reported problems smelling, had developed Alzheimer's disease by follow-up (n.s.).

Low olfaction scores $(\leq 34)$ predicted the diagnosis of Alzheimer's disease at follow-up (19 of 47 with low olfaction scores developed Alzheimer's disease compared to zero of 30 with high olfaction scores $)\left(\chi^{2}=16.1, \mathrm{df}=1\right.$, $\mathrm{p}<0.001)$; all 19 patients with mild cognitive impairment who developed Alzheimer's disease had low olfaction scores. Low olfaction scores accompanied by subjective report of no problems smelling were present in 16 of 19 patients who met the criteria for Alzheimer's disease at follow-up compared to 21 of 58 who did not meet (or had not yet met) the criteria for Alzheimer's disease at follow-up $\left(\chi^{2}=13.2, \mathrm{df}=1, \mathrm{p}<0.001\right)$. When we examined cutoff points 
FIGURE 1. Kaplan-Meier Survival Curve of Probability of Developing Alzheimer's Disease Over 4 Years in Subjects With Mild Cognitive Impairment at Baseline, by Presence or Absence of Baseline Olfactory Deficit Plus Lack of Awareness of Deficit

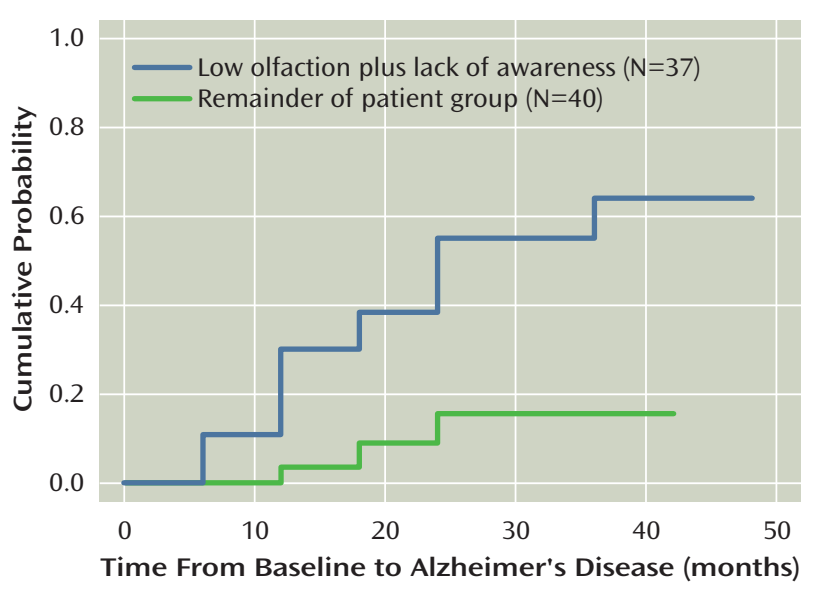

for "low olfaction score" across the $30-36$ ( $\leq 30$ to $\leq 36$ ) scoring range, low olfaction plus lack of awareness remained a significant predictor of Alzheimer's disease $\left(\chi^{2}=\right.$ 8.9-13.2, df $=1, \mathrm{p}<0.01-0.001$ ). Fourteen of 19 who developed Alzheimer's disease had low olfaction plus lack of awareness at a cutoff point of $\leq 33,16$ of 19 who developed Alzheimer's disease had low olfaction plus lack of awareness at a cutoff point of $\leq 35$ (or $\leq 34$; cutoff used in the analyses), and 16 of 19 who developed Alzheimer's disease had low olfaction plus lack of awareness at a cutoff point of $\leq 36$. Among patients with mild cognitive impairment, only three $(3.9 \%)$ of 77 reported problems smelling but scored $\geq 35$ on the olfaction test.

The Kaplan-Meier survival curve for patients with low olfaction plus lack of awareness, compared to that for the remainder of the patient group, is presented in Figure 1. In a Cox proportional hazards model, olfaction scores alone predicted time to develop Alzheimer's disease $\left(\chi^{2}=8.8, \mathrm{df}=\right.$ $1, \mathrm{p}<0.005$ ), but subjective reports of problems smelling analyzed alone were not predictive of Alzheimer's disease. In Cox analyses, low olfaction scores (or olfaction scores dichotomized as $\leq 34$ versus $>34$ ) were not significantly predictive when age (n.s.), sex (n.s.), modified Mini-Mental State score (n.s.), and years of education (n.s.) were entered into the same model. Low olfaction plus lack of awareness was a significant predictor (relative risk $=7.3$, $95 \%$ confidence interval $[C I]=1.7-23.1, \mathrm{p}<0.01$ ) of time to develop Alzheimer's disease when age (n.s.), sex (n.s.), modified Mini-Mental State score (n.s.), and years of education (n.s.) were also included in the Cox model. This effect remained when, instead of modified Mini-Mental State score, the attention (Target Finding Test letter cancellation task) or memory (Selective Reminding Test total recall task) measures were included in this model with low olfaction plus lack of awareness (attention: relative risk= 10.7, 95\% CI=2.5-41.0, $\mathrm{p}<0.005$; and memory: relative risk $=4.7,95 \% \mathrm{CI}=1.2-18.2, \mathrm{p}<0.05)$. Low olfaction plus lack of awareness remained a significant predictor of time until the development of Alzheimer's disease (relative risk=7.3, 95\% CI=1.8-42.7, $\mathrm{p}<0.01$ ), even after entering age (n.s.), sex (n.s.), and all three neuropsychological measures (modified Mini-Mental State score [n.s.], attention measure $[\mathrm{p}<0.02]$, and memory measure [n.s.]) into the same Cox model.

For each olfaction score in the 31-36 range, the low olfaction plus lack of awareness variable remained a significant predictor of time to develop Alzheimer's disease in Cox analyses that included age, sex, years of education, and modified Mini-Mental State score as covariates. For an olfaction score of $\leq 30$, the values for low olfaction plus lack of awareness were not significant.

Additional Cox analyses were conducted separately in the subgroup with high baseline Mini-Mental State scores ( $\geq 27$ of $30, N=52$ ); low olfaction plus lack of awareness remained a significant predictor of time until the development of Alzheimer's disease (relative risk $=10.8,95 \% \mathrm{CI}=$ $1.1-105.0, \mathrm{p}<0.05)$ when age, sex, years of education, and baseline Mini-Mental State score were included in the model. In this subgroup with high scores on the MiniMental State, eight of nine patients who met the criteria for Alzheimer's disease had baseline low olfaction plus lack of awareness ratings compared to 15 of 43 who did not meet (or had not yet met) the criteria for Alzheimer's disease $\left(\chi^{2}=8.8, \mathrm{df}=1, \mathrm{p}<0.01\right)$. The nine patients with high Mini-Mental State scores who were diagnosed with Alzheimer's disease at the 2-year follow-up had significantly lower baseline olfaction scores (mean=27.1, SD=5.9) than the 43 patients who were not diagnosed with Alzheimer's disease at the 2-year follow-up (mean=34.1, $\mathrm{SD}=4.6)(\mathrm{t}=$ 3.8, $\mathrm{df}=51, \mathrm{p}<0.001)$.

To evaluate clinically relevant prediction in the intermediate term, further analyses were conducted by restricting the clinical group with mild cognitive impairment to the patients who had completed 2 years of follow-up $(\mathrm{N}=36)$ or had already developed Alzheimer's disease by 2 years $(\mathrm{N}=4)$. In this subsample of 40 patients with mild cognitive impairment (18 who had and 22 who had not developed Alzheimer's disease), baseline olfaction scores were lower in those who had developed Alzheimer's disease (mean= 26.3, $\mathrm{SD}=7.7$ ) than in those who had not (mean=31.5, $\mathrm{SD}=$ 7.4) $(\mathrm{t}=2.1, \mathrm{df}=38, \mathrm{p}<0.05)$. Fifteen of 18 patients who met the criteria for Alzheimer's disease at the 2-year follow-up had low olfaction plus lack of awareness at baseline compared to nine of 22 who had not met (or have not yet met) the criteria for Alzheimer's disease $\left(\chi^{2}=7.4, \mathrm{df}=1, \mathrm{p}<0.01\right)$. In survival analyses that evaluated the outcome of Alzheimer's disease at the 2-year follow-up, low baseline olfaction scores (or olfaction scores dichotomized as $\leq 34$ versus $>34$ ) did not predict Alzheimer's disease when age, sex, modified Mini-Mental State score, and years of education were also included in the model. However, low olfaction plus lack of awareness at baseline was a significant predic- 
tor of Alzheimer's disease (relative risk $=6.4,95 \% \mathrm{CI}=1.5-$ 26.8, $\mathrm{p}<0.01$ ) when age, sex, modified Mini-Mental State score, and years of education were also included in the model. Similarly, for patients who had completed the 2year follow-up, logistic regression analyses revealed that low olfaction plus lack of awareness at baseline was a significant predictor of Alzheimer's disease (odds ratio=13.1, 95\% CI=1.6-116.0, p<0.05) when age, sex, modified MiniMental State score, and years of education were also included in the model.

For this subgroup followed up at 2 years, baseline olfaction total scores of $\leq 34$ led to $100 \%$ sensitivity and $45.5 \%$ specificity for the diagnosis of Alzheimer's disease, including a progressive decrease in sensitivity and an increase in specificity with lower olfaction scores (Table 1). Comparable figures were obtained for the low olfaction plus lack of awareness variable by using a wide range of cutoff points for "low olfaction score" (Table 1). For both olfaction variables, the optimal tradeoff between sensitivity and specificity appeared to be in the 30-35 scoring range.

The apolipoprotein E genotype was evaluated in 77 patients with mild cognitive impairment. Only 11 (14.3\%) of the 77 patients with mild cognitive impairment had the apolipoprotein E $\varepsilon 4$ allele (hetero- or homozygous), and four of these 11 patients had developed Alzheimer's disease. There was no association between the presence of the $\varepsilon 4$ allele and either of the olfaction measures or the outcome of Alzheimer's disease, but the small number of patients with the $\varepsilon 4$ allele precluded meaningful statistical analyses.

\section{Discussion}

In this clinical group of patients with mild cognitive impairment, low olfactory identification test scores at baseline predicted the diagnosis of Alzheimer's disease at follow-up. Subjective reports of no problems smelling were not predictive of the development of Alzheimer's disease because most patients who reported no problems smelling actually scored well ( $\geq 35$ of 40 ) on the olfaction test (like most of the general population). However, 16 of 19 patients who reported no problems smelling yet scored low on the olfaction test had developed Alzheimer's disease by the 2-year follow-up. This effect remained significant in Cox survival analyses after controlling for age, sex, years of education, and cognitive scores (on the modified Mini-Mental State and attention or memory measures), indicating that the results could not be explained by lack of attention or poor memory. Similar findings were obtained by using logistic regression analyses for the subgroup followed up at 2 years. Although low olfaction score alone predicted time to develop Alzheimer's disease, it did not predict time to develop Alzheimer's disease in Cox analyses that controlled for age, sex, years of education, and modified Mini-Mental State score, indicating overlapping variance in prediction (baseline olfaction score cor-
TABLE 1. Sensitivity and Specificity of Olfaction and Olfaction Plus Lack of Awareness of Olfactory Deficit in Predicting Alzheimer's Disease at 2-Year Follow-Up ${ }^{a}$ in 40 Patients With Mild Baseline Cognitive Impairment ${ }^{\text {b }}$

\begin{tabular}{|c|c|c|c|c|}
\hline \multirow{2}{*}{$\begin{array}{l}\text { Cutoff for } \\
\text { Olfaction Score } \\
\text { (range }=0-40 \text { ) }\end{array}$} & \multicolumn{2}{|c|}{$\begin{array}{l}\text { Baseline Olfaction } \\
\text { Score }\end{array}$} & \multicolumn{2}{|c|}{$\begin{array}{l}\text { Olfaction Score Plus } \\
\text { Lack of Awareness } \\
\text { of Olfactory Deficit }\end{array}$} \\
\hline & $\begin{array}{l}\text { Sensitivity } \\
(\%)\end{array}$ & $\begin{array}{c}\text { Specificity } \\
(\%)\end{array}$ & $\begin{array}{c}\text { Sensitivity } \\
(\%)\end{array}$ & $\begin{array}{c}\text { Specificity } \\
(\%)\end{array}$ \\
\hline$<27$ & 44.4 & 81.8 & 27.8 & 86.4 \\
\hline$<28$ & 44.4 & 81.8 & 27.8 & 86.4 \\
\hline$<29$ & 44.4 & 77.3 & 27.8 & 81.8 \\
\hline$<30$ & 55.6 & 77.3 & 38.9 & 81.8 \\
\hline$<31$ & 55.6 & 72.7 & 38.9 & 81.8 \\
\hline$<32$ & 61.1 & 68.2 & 44.4 & 77.2 \\
\hline$<33$ & 77.8 & 59.1 & 61.1 & 72.7 \\
\hline$<34$ & 88.9 & 50.0 & 72.2 & 63.6 \\
\hline$<35$ & 100.0 & 45.5 & 83.3 & 59.1 \\
\hline$<36$ & 100.0 & 36.4 & 83.3 & 54.5 \\
\hline$<37$ & 100.0 & 18.1 & 83.3 & 36.4 \\
\hline
\end{tabular}

${ }^{a}$ Four patients had not yet been followed up for the full 2 years but had already developed Alzheimer's disease.

${ }^{b}$ Olfaction was measured with the University of Pennsylvania Smell Identification Test. The test's first question, "Do you suffer from smell problems?," was used to determine awareness of olfactory problems. Subjects who answered "no" and had olfaction scores $\leq 34$ were considered to lack awareness of the olfactory deficit.

related significantly with age [inverse], education, and modified Mini-Mental State score). The results suggest that low olfaction plus lack of awareness may not have the same degree of overlapping variance with these demographic and clinical factors and, hence, may contribute uniquely to the prediction of time to develop Alzheimer's disease.

In patients with high baseline scores on the Mini-Mental State ( $\geq 27$ of 30), low olfaction plus lack of awareness remained a significant predictor of Alzheimer's disease in Cox analyses that controlled for demographic and clinical factors. This suggests the possible predictive utility of olfactory deficits in patients with minimal cognitive deficits, who are often difficult to diagnose and in whom prognosis is unclear. For the 2-year prediction of Alzheimer's disease, both the low olfaction and low olfaction plus lack of awareness variables showed high sensitivity for scores of $\leq 35$, with high specificity for scores of $\leq 30$. Scores above or below the 30-35 range led to relatively low sensitivity or specificity (Table 1), suggesting that University of Pennsylvania Smell Identification Test scores in the 30-35 range may be useful in patients with mild cognitive impairment as a potential predictor of Alzheimer's disease. In crosssectional studies that compared patients with Alzheimer's disease to comparison subjects and first-degree relatives of Alzheimer's patients to comparison subjects, cutoff scores of 27 and 30 on the University of Pennsylvania Smell Identification Test showed the best discrimination, respectively $(7,9)$. Olfaction scores are known to decrease with increasing severity of the disease (7), so it is not surprising that the cutoff scores for the University of Pennsylvania Smell Identification Test showing the best prediction of Alzheimer's disease in our study of mild cognitive 
impairment were higher than those in studies of patients with Alzheimer's disease.

In our study group, sample data were used to derive the optimal cutoff scores $(\leq 34)$, as was done in studies of patients with Alzheimer's disease (7,9). Given this limitation and the paucity of other data on the use of the University of Pennsylvania Smell Identification Test in predicting Alzheimer's disease in patients with mild cognitive impairment, our findings require independent replication before specific cutoff scores on the University of Pennsylvania Smell Identification Test can be recommended for use in clinical practice. Also, linking low olfaction scores with the symptom of lack of awareness of the olfactory deficit to create a dichotomous variable has not been done before, to our knowledge, in studies of mild cognitive impairment or Alzheimer's disease, further emphasizing the need for independent replication.

One consequence of the follow-up being limited to date is that these analyses absorb considerable error because many current patients with mild cognitive impairment but without Alzheimer's disease at the 2-year follow-up will develop Alzheimer's disease after longer follow-up intervals. The use of survival analyses only partly addressed this limitation, which precludes being definitive about the prediction of long-term outcomes. Another limitation of the study was the use of a single "yes" or "no" item for subjective reports of problems smelling. Future research should evaluate in greater depth this subjective symptom, which, to our knowledge, has not previously been evaluated in patients with mild cognitive impairment.

We found that patients with mild cognitive impairment scored lower than matched normal comparison subjects on the University of Pennsylvania Smell Identification Test. This result is consistent with findings from other studies that compared patients with mild cognitive impairment (5) and patients in the early stages of Alzheimer's disease (2-4) to normal comparison subjects. The findings with the low olfaction plus lack of awareness variable are also consistent with results from a study that reported lack of awareness of loss of smell in $74 \%$ of the patients with Alzheimer's disease compared to only $8 \%$ of the patients with sinusitis (36). Other studies have shown that unlike olfaction deficits, deficits in other sensory modalities, including taste, do not distinguish patients with Alzheimer's disease from normal comparison subjects $(3,5)$.

From a theoretical perspective, lack of awareness of the olfactory deficit can be considered a type of anosognosia. In the patients with mild cognitive impairment who subsequently met the criteria for Alzheimer's disease, anosognosia was in the domain of lack of awareness of the olfactory deficit and not in the domain of lack of awareness of the cognitive deficit; the latter was a likely artifact of the fact that this was a clinical group with mild cognitive impairment that was evaluated for cognitive complaints. Anosognosia, broadly defined, is thought to be mediated primarily by the parietal lobe (37), although the frontal lobe may also be involved (38). In Anton's syndrome, unawareness of the visual deficit is due to damage to the visual association cortex, which is in close proximity to the primary visual cortex (39). Using this analogy, we speculate that awareness of the loss of the sense of smell, for which the brain center remains unidentified, may be localized to medial-temporal lobe structures that are known to be affected early in Alzheimer's disease and are associated with olfactory detection deficits (10). This could explain why low olfaction scores accompanied by a lack of awareness of the olfactory deficit in patients with mild cognitive impairment strongly predicted Alzheimer's disease at follow-up.

In patients with Alzheimer's disease, both a sensory deficit and a higher-level deficit in the cortical perception and integration of an olfactory stimulus may occur (10). The findings of deficits in both olfactory detection and the awareness of olfactory deficits suggest that deficits at both the sensory level and the level of higher-order processing for olfaction occur at the stage of mild cognitive impairment in patients who eventually meet the clinical diagnostic criteria for Alzheimer's disease. The strength of our findings suggests potential clinical utility for olfactory deficits, particularly the variable for low olfaction plus lack of awareness, as an early diagnostic marker of Alzheimer's disease. Longer follow-up periods and independent replication in larger clinical groups are needed to establish clinical utility.

Received July 12, 1999; revisions received Nov. 29, 1999, and March 28, 2000; accepted April 25, 2000. From the Memory Disorders Clinic, Taub Alzheimer's Disease Research Center, New York State Psychiatric Institute; and the Department of Psychiatry, the Department of Neurology, the Department of Public Health, and the Gertrude H. Sergievsky Center, College of Physicians and Surgeons, Columbia University, New York. Address reprint requests to Dr. Devanand, New York State Psychiatric Institute, 722 West 168th St., New York, NY 10032.

Supported in part by NIMH grants $\mathrm{MH}-50038$ and $\mathrm{MH}-44176$; NIH grants AG-07370, AG-08702, and AG-10963 from the National Institute on Aging; NIH grant RR-00645 from the Division of Research Resources; and a grant from the Charles S. Robertson Memorial Gift for Research in Alzheimer's Disease.

\section{References}

1. Talamo BR, Rudel R, Kosik KS, Lee VM, Neff S, Adelman L, Kauer JS: Pathological changes in olfactory neurons in patients with Alzheimer's disease. Nature 1989; 337:736-739

2. Doty RL, Reyes PF, Gregor T: Presence of both odor identification and detection deficits in Alzheimer's disease. Brain Res Bull 1987; 18:597-600

3. Murphy C, Gilmore MM, Seery CS, Salmon DP, Lasker BR: Olfactory thresholds are associated with degree of dementia in Alzheimer's disease. Neurobiol Aging 1990; 11:465-469

4. Morgan CD, Nordin S, Murphy C: Odor identification is an early marker for Alzheimer's disease: impact of lexical functioning and detection sensitivity. J Clin Exp Neuropsychol 1995; 17: 793-803

5. Nordin S, Murphy C: Impaired sensory and cognitive olfactory function in questionable Alzheimer's disease. Neuropsychology 1996; 10:113-119 
6. Murphy C, Nordin S, Acosta L: Odor learning, recall, and recognition memory in young and elderly adults. Neuropsychology 1997; 11:126-137

7. Serby M, Larson P, Kalkstein D: The nature and course of olfactory deficits in Alzheimer's disease. Am J Psychiatry 1991; 148: 357-360

8. Nordin S, Almkvist O, Berglund B, Wahlund L: Olfactory disfunction for pyridine and dementia progression in Alzheimer disease. Arch Neurol 1997; 54:993-998

9. Serby M, Mohan C, Aryan M, Williams L, Mohs RC, Davis KL: Olfactory identification deficits in relatives of Alzheimer's disease patients. Biol Psychiatry 1996; 39:375-377

10. Martzke JS, Kopala LC, Good KC: Olfactory dysfunction in neuropsychiatric disorders: review and methodological considerations. Biol Psychiatry 1997; 42:721-732

11. Doty RL, Perl DP, Steele JC, Chen KM, Pierce JD, Reyes P, Kurland LT: Olfactory dysfunction in three neurodegenerative diseases. Geriatrics 1991; 46:47-51

12. Warner M, Peabody C, Csernansky J: Olfactory functioning in schizophrenia and depression. Biol Psychiatry 1990; 27:457467

13. Sajjadian A, Doty RL, Gutnick DN, Chirugi RJ, Sivak M, Perl D: Olfactory dysfunction in amyotrophic lateral sclerosis. Neurodegeneration 1994; 3:153-157

14. Mesholam RI, Moberg PJ, Mahr RN, Doty RL: Olfaction in neurodegenerative disease: a meta-analysis of olfactory functioning in Alzheimer's and Parkinson's diseases. Arch Neurol 1998; 55:84-90

15. Devanand DP, Folz M, Gorlyn M, Moeller JR, Stern Y: Questionable dementia: clinical course and predictors of outcome. J Am Geriatr Soc 1997; 45:321-328

16. Albert SM, Michaels K, Padilla M, Pelton G, Bell K, Marder K, Stern Y, Devanand DP: Functional significance of mild cognitive impairment in elderly patients without a dementia diagnosis. Am J Geriatr Psychiatry 1999; 7:213-220

17. Hughes CP, Berg L, Danzinger WL, Coben LA, Martin RL: A new clinical scale for the staging of dementia. Br J Psychiatry 1982; 140:566-572

18. Mayeux R, Stern Y, Rosen J, Leventhal J: Depression, intellectual impairment and Parkinson's disease. Neurology 1981; 31:645650

19. Folstein MF, Folstein SE, McHugh PR: "Mini-Mental State": a practical method for grading the cognitive state of patients for the clinician. J Psychiatr Res 1975; 12:189-198

20. Buschke H, Fuld AP: Evaluating storage, retention, and retrieval in disordered memory and learning. Neurology 1974; 24:1019-1025

21. Petersen RC, Smith GE, Ivnik R, Tangalos EG, Schaid DJ, Thibodeau SN, Kokman E, Waring SC, Kurland LT: Apolipoprotein E status as a predictor of the development of Alzheimer's disease in memory-impaired individuals. JAMA 1995; 273:12741278
22. Tierney MC, Szalai JP, Snow WG, Fisher RH, Nores A, Nadon G, Dunn E, St George-Hyslop PH: Prediction of probable Alzheimer's disease in memory-impaired patients: a prospective longitudinal study. Neurology 1996; 46:661-665

23. Wechsler D: The Wechsler Memory Scale. San Antonio, Tex, Psychological Corp (Harcourt), 1990

24. Buschke H, Fuld PA: Evaluating storage, retention, and retrieval in disordered memory and learning. Neurology 1974; 24:1019-1025

25. Rosen WG: Rosen Drawing Test. Bronx, NY, Department of Veterans Affairs Medical Center, 1981

26. Benton A: Neurosensory Center Comprehensive Examination for Aphasia. Edited by Spreen O and Benton A. Victoria, BC, Canada, University of Victoria Press, 1967

27. Goodglass H, Kaplan D: The Assessment of Aphasia and Related Disorders, 2nd ed. Philadelphia, Lea \& Febiger, 1983

28. Kaplan E, Goodglass H, Weintraub S: The Boston Naming Test, 2nd ed. Philadelphia, Lea \& Febiger, 1983

29. Benton A: The Visual Retention Test. New York, Psychological Corporation, 1955

30. Sano M, Rosen W, Mayeux R: Attention Deficits in Alzheimer's Disease. New York, American Psychological Association, 1984

31. McKhann G, Drachman D, Folstein M, Katzman R, Price D, StadIan EM: Clinical diagnosis of Alzheimer's disease: report of the NINCDS-ADRDA Work Group under the auspices of the Department of Health and Human Services Task Force on Alzheimer's Disease. Neurology 1984; 34:939-944

32. Devanand DP, Brockington CD, Moody BJ, Brown RP, Mayeux R, Endicott J, Sackeim HA: Behavioral syndromes in Alzheimer's disease. Int Psychogeriatr 1992; 4:161-184

33. Hixson J, Vernier D: Restriction isotyping of human apolipoprotein $\mathrm{E}$ by gene amplification and cleavage with HhA1. J Lipid Res 1991; 31:545-548

34. Doty RL, Newhouse MG, Azzalina JD: Internal consistency and short-term test-retest reliability of the University of Pennsylvania Smell Identification Test. Chem Senses 1985; 10:297-300

35. Blessed G, Tomlinson BE, Roth M: The association between quantitative measures of dementia and of senile change in the cerebral grey matter of elderly subjects. Br J Psychiatry 1982; 140:566-572

36. Nordin S, Monsch AU, Murphy C: Unawareness of smell loss in normal aging and Alzheimer's disease: discrepancy between self-reported and diagnosed smell sensitivity. J Gerontol 1995; 50B:187-192

37. Lopez OL, Becker JT, Somsak D, Dew MA, DeKosky ST: Awareness of cognitive deficits and anosognosia in probable Alzheimer's disease. Eur Neurol 1994; 34:277-282

38. Starkstein SE, Vasquez S, Miglioretti R, Teson A, Sabe L, Leiguarda R: A single-photon emission computed tomographic study of anosognosia in Alzheimer's disease. Arch Neurol 1995; 52:415-420

39. Ramachandran VS: Anosognosia in parietal lobe syndrome. Conscious Cogn 1995; 4:22-51 